DOI: $10.5455 / 2320-1770 . i j \operatorname{cog} 20130956$

\title{
Pyocolpos in a 4 year old: a rare scenario
}

\author{
Neha Agarwal $^{1 *}$, Saroj Singh ${ }^{1}$, Rajeshwar Dayal ${ }^{2}$, Anu Pathak ${ }^{1}$
}

${ }^{1}$ Department of Obstetrics and Gynecology, ${ }^{2}$ Department of Pediatrics, S.N. Medical College, Agra, U.P., India

Received: 28 July 2013

Accepted: 4 August 2013

\author{
*Correspondence: \\ Dr. Neha Agarwal, \\ E-mail: its_my_ishtyle@yahoo.com
}

(C) 2013 Agarwal $\mathrm{N}$ et al. This is an open-access article distributed under the terms of the Creative Commons Attribution Non-Commercial License, which permits unrestricted non-commercial use, distribution, and reproduction in any medium, provided the original work is properly cited.

\begin{abstract}
Pyocolpos is cystic dilatation of vagina due to accumulation of pus resulting from genital tract obstruction. Hydrocolpos or pyocolpos are usually encountered either in the neonatal period due to stimulation from maternal hormones or at menarche. Their occurrence beyond infancy and before puberty is rare. We report this rare case of pyocolpos in a 4 year old child.
\end{abstract}

Keywords: Hydrocolpos, Imperforate hymen, Pyocolpos

\section{INTRODUCTION}

Pyocolpos may be defined as 'a congenital atresia of the vaginal orifice, with retention of an excess of secondarily infected cervical secretions'., ${ }^{1,2}$ The main causes of obstruction are imperforate hymen, transverse vaginal membrane or vaginal atresia. ${ }^{3}$ Pyocolpos is rare at any age and all cases should be reported as the treatment is simple when once the condition has been recognized.

\section{CASE REPORT}

A 4 year old girl presented to the pediatric outpatient clinic with complaints of fever followed by excessive vaginal discharge for 4-5 days. There was history of repeated attacks of fever for the last 2-3 months. There was no history of pain or distension of abdomen, no difficulty in passing urine or feces and no history of associated itching or burning. There was no family history of diabetes. She was admitted there and on showing no response to parenteral antibiotics, referred to Gynecological ward. History of any local trauma or foreign body insertion was excluded. Any history suggestive of immune-compromised status was excluded.

On examination, the child appeared to be in fair general condition. There was no abdominal swelling. On local examination, excoriation was present at vulva and inner aspect of both thighs. Purulent fluid was leaking from the introitus. Detailed examination could not be done at this stage as child was very irritable. Her hemoglobin was 8.3 $\mathrm{g} \%$, total leucocyte count $16000 / \mathrm{mm}^{3}$ with $84 \%$ polymorphs. Urine showed $>100$ pus cells/hpf, which were gradually reduced to 50 with antibiotics. Urine culture was sterile as she was already on antibiotics. Her renal function test and liver function tests were normal. $\mathrm{HIV}$ and HBsAg status was negative.

As seen in Figures 1A and B, ultrasound showed a large thick walled cystic lesion, measuring $9.6 \times 5.8 \mathrm{~cm}$, seen posterior to urinary bladder (in the line of vagina). Low level internal echoes were present in the cystic lesion. The uterine body was seen pushed up with a small amount of fluid collection in the uterine cavity too. Both the kidneys, ureters and urinary bladder were normal.

She was admitted and prepared for evacuation of vaginal pus under anesthesia. Per-operative genital examination showed an imperforate hymen with purulent fluid leaking from one side of the hymenand normally placed urethra and anus. The contents were drained vaginally by extending the opening by a small transverse incision which revealed purulent fluid draining from the dilated vagina. Vaginal epithelium was stitched to hymenal ring 
by four interrupted absorbable sutures. The drained fluid was sent for culture and sensitivity which was sterile at that time. Antibiotics were prescribed for another few days. The patient showed good clinical response.
Postoperative follow up sonogram revealed normal vaginal echoes with no cystic collection and normally positioned uterus with normal endometrial echoes (Figures 2A and B).

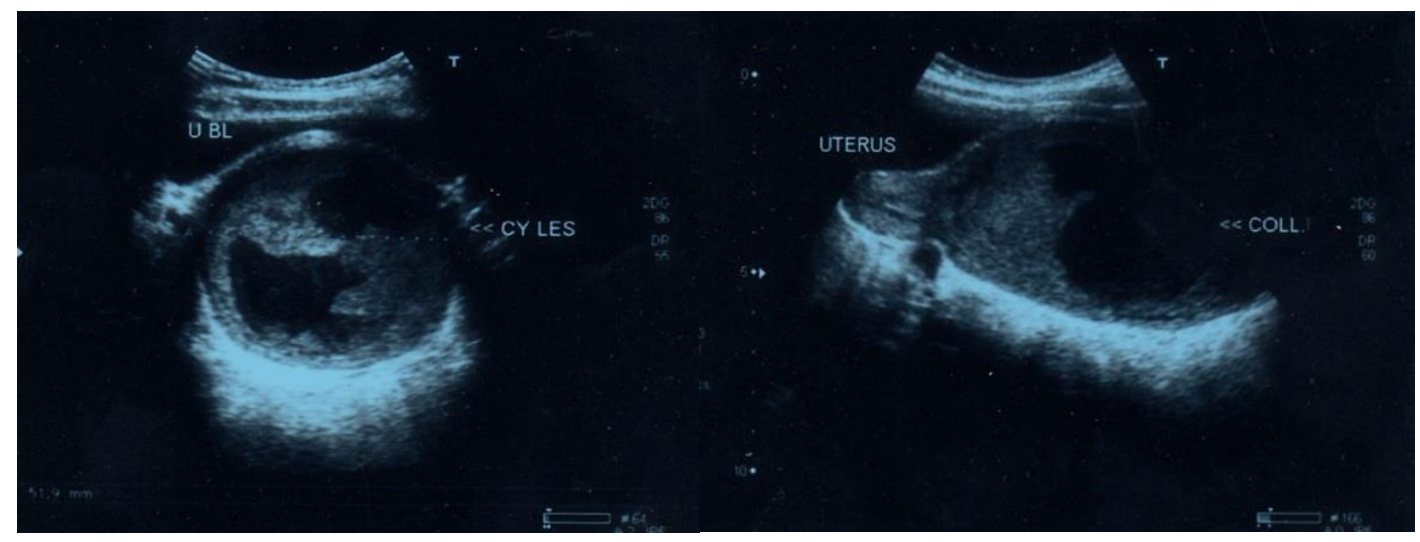

Figure 1: (A) Ultrasound picture showing a large thick walled cystic lesion $(9.6 \times 5.8 \mathrm{~cm})$ posterior to urinary bladder with low level internal echoes, (B) The uterine body is pushed up with a small amount of fluid collection in the cavity.

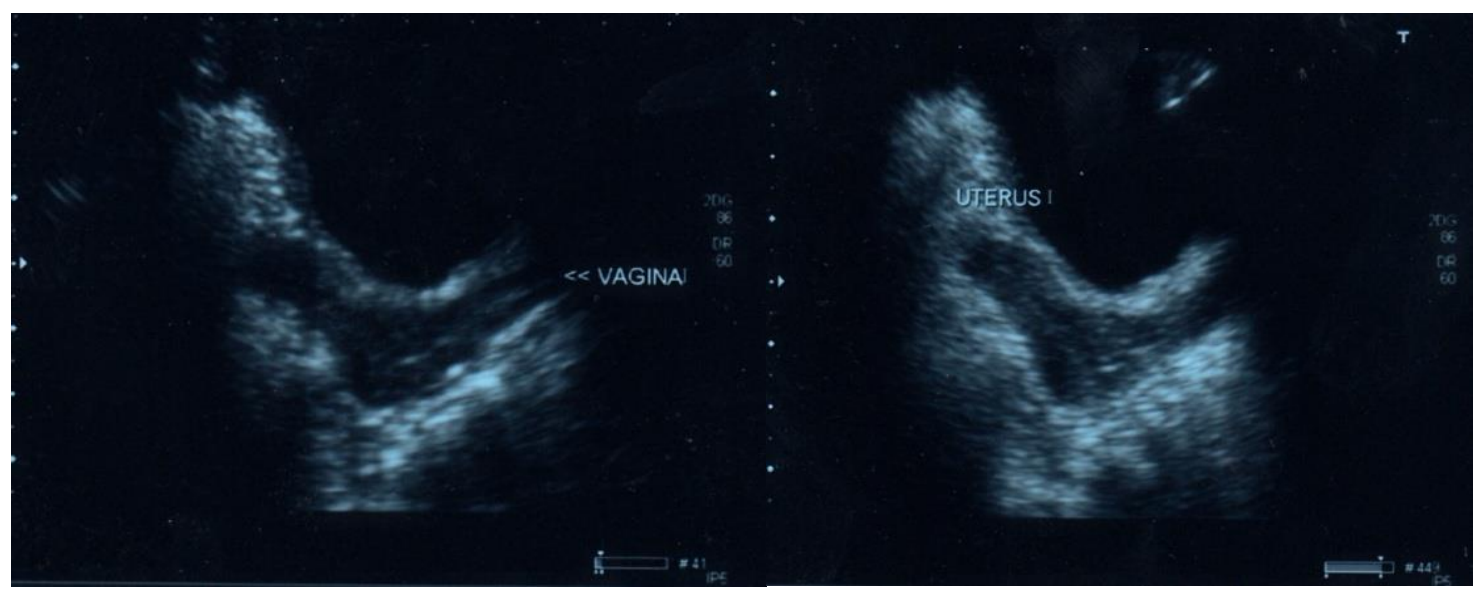

Figures 2: Postoperative follow up sonogram- (A) showing normal vaginal echoes with no cystic collection and (B) normally positioned uterus with normal endometrial echoes.

\section{DISCUSSION}

Hydrocolpos or pyocolpos is a rare congenital disorder with prevalence of 1 in 30,000 births. ${ }^{4}$ Very few cases of pyocolpos have been reported in literature. Brevetti et al reported pyocolpos in a 3 month old associated with urinary tract infection. ${ }^{5}$ Pascaud et al reported a similar case of a $41 / 2$ years old girl found to have secondarily infected retained secretions in the vagina. However, it resulted from a vaginal membrane not imperforate hymen. ${ }^{6}$

Clinical symptoms of pyocolposare usually insidious, unless it becomes an evident mass in the lower abdomen or when it compresses the ureter and causes hydronephrosis or a urinary tract infection. In our case the pyocolposdid not cause pressure symptoms of the urinary tract or gastrointestinal system, but due to the pressure, the pus found its way out rupturing the hymen.

\section{CONCLUSION}

Disorders from imperforate hymen are rarebeyond infancy and before puberty, mainly because of the scantiness of uterine and vaginal secretions during childhood. Hydrocolposoccurs due to accumulation of fluid behind the occluded orifice and its secondary infection leads to pyocolpos. Delayed surgery due to late detection is common and can result in major risk due to infection, pressure symptoms, sepsis and associated 
congenital anomalies. Thus, early detection by genital examination and imaging is required to prevent complications.

\section{REFERENCES}

1. Badeli H, Mircharkhcian M, Pirooz A. Prolonged sterile dysuria as a first presentation of Pyocolpos. IJKD 2010;4:82-4.

2. Todd IP. Pyocolpos in infancy. Arch Dis Child. 1946 June; 21(106):118-9.

3. Nazir Z, Rizvi RM, Qureshi RN. Congenital vaginal obstruction: varied presentation and outcome. Pediatr Surg Int 2006;22:749-53.
4. Gandage SG, Aironi VD, Agarwal S, Singh J. Ultrasound diagnosis of pyocolpos. J Indian Assoc Pediatr Surg 2007;12(4):235-7.

5. Brevetti LS, Kimura K, Brevetti GR, Lawrence JP, Soper RT. Pyocolpos: diagnosis and treatment. J Pediatr Surg 1997 Jan;32(1):110-1.

6. Pascaud E, Hugon J, Pascaud JL, Rousseau J. Vaginal membrane with hydrocolpos and pyocolpos in children. J Radiol Electrol Med Nucl. 1979 Mar;60(3):215-9.

DOI: $10.5455 / 2320-1770 . i j \operatorname{cog} 20130956$

Cite this article as: Agarwal N, Singh S, Dayal R, Pathak A. Pyocolpos in a 4 year old: a rare scenario. Int J Reprod Contracept Obstet Gynecol 2013;2:488-90. 